\title{
COMMENT AND REVIEWS
}

\author{
THE MULTIPLE MUTATION IN WHEAT
}

(I) If, as Lein suggests (Heredity, 5, 147-149), varieties of Triticum vulgare carry a considerable load of $s v$ mutant genes, is it likely that no sv phenotypes should have been seen, striking as they are? Yet, as mentioned in my paper, none are on record. The three varieties concerned are respectively of North-Western European, Western Mediterranean, and Canadian (originally Eastern European) origin. Hence sv mutations would be either of ancient origin, or of high frequency; one of them is supposed to have arisen in the breeding experiment itself. Why is it then that not in one of the many thousands of wheat crosses made in the last sixty years or more should a so phenotype have occurred either by segregation or by mutation?

(2) On the evidence produced in my paper, the mutant arose as a homozygote. This, according to Lein's assumption, requires not one mutation step, as he suggests, but two, viz., one in each allele of one gene.

(3) The deletion postulated by Lein need not, as suggested in his final sentence, be one of the entire BC arm ; unevenly broken bridges supply deletions with a high frequency.

O. H. Frankel.

HEREDITARY GENIUS. By Francis Galton. London : Watts \& Co. Second edition reprinted 1950. Pp. Xxvii+379. 10s. 6d.

Just half a century has now elapsed since the Mendelian renaissance of I9oo. The early promise of Mendelian genetics has been richly fulfilled and vast contributions have been made both to our theoretical understanding and to the solution of practical problems. In the sphere of human genetics important applications include the use of blood groups in paternity testing and in predicting the risk of hæmolytic disease of the newborn; also the use of knowledge about the inheritance of a variety of diseases and abnormalities both as an aid to clinical diagnosis and for purposes of eugenic prognosis. It is important to realise, however, that the greatest successes have been obtained with characters which are mainly controlled by single genes, or at most small numbers of genes. Thus the greater part of human genetics is at present concerned with characters whose practical repercussions, though of considerable importance, affect relatively few individuals.

It was Francis Galton who first effectively demonstrated that not only were quantitative characters like stature subject to heredity, but so also were intelligence, fertility and other more subtle and less easily quantified aspects of the human spirit. Galton's use of human data with its small families and doubtful ancestries, together with the emphasis on quantitative characters prevented a satisfactory understanding of the actual laws of inheritance. The statistical approach, initiated by Galton and further developed by Karl Pearson, produced a distinct school of biometricians. There was at first great rivalry and even antagonism between the 